\title{
IL-17RD (Sef or IL-17RLM) interacts with IL-17 receptor and mediates IL-17 signaling
}

Zhili Rong ${ }^{1}$, Anan Wang ${ }^{2}$, Zhiyong $\mathrm{Li}^{1}$, Yongming Ren ${ }^{1}$, Long Cheng ${ }^{1}$, Yinghua $\mathrm{Li}^{1}$, Yinyin Wang ${ }^{1}$, Fangli Ren ${ }^{1}$, Xiaoning Zhang ${ }^{1}$, Jim $\mathrm{Hu}^{2}$, Zhijie Chang ${ }^{1}$

${ }^{1}$ School of Medicine, Department of Biological Sciences and Biotechnology, State Key Laboratory of Biomembrane and Membrane Biotechnology, Tsinghua University, Beijing 100084, China; ${ }^{2}$ Department of Laboratory Medicine and Pathobiology, Physiology and Experimental Medicine, Hospital for Sick Children Research Institute, University of Toronto, Toronto, Canada M5G $1 X 8$

Interleukin-17 (IL-17 or IL-17A) production is a hallmark of $\mathrm{T}_{\mathrm{H}} 17$ cells, a new unique lineage of $\mathrm{CD}^{+} \mathrm{T}_{\text {lympho- }}$ cytes contributing to the pathogenesis of multiple autoimmune and inflammatory diseases. IL-17 receptor (IL-17R or IL-17RA) is essential for IL-17 biological activity. Emerging data suggest that the formation of a heteromeric and/or homomeric receptor complex is required for IL-17 signaling. Here we show that the orphan receptor IL-17RD (Sef, similar expression to FGF genes or IL-17RLM) is associated and colocalized with IL-17R. Importantly, IL-17RD mediates IL-17 signaling, as evaluated using a luciferase reporter driven by the native promoter of $24 \mathrm{p} 3$, an IL-17 target gene. In addition, an IL-17RD mutant lacking the intracellular domain dominant-negatively suppresses IL-17Rmediated IL-17 signaling. Moreover, IL-17RD as well as IL-17R is associated with TRAF6, an IL-17R downstream molecule. These results indicate that IL-17RD is a part of the IL-17 receptor signaling complex, therefore providing novel evidence for IL-17 signaling through a heteromeric and/or homomeric receptor complex.

Keywords: IL-17RD, sef, IL-17, IL-17R, IL-17 signaling, heteromeric receptor complex, $\mathrm{T}_{\mathrm{H}} 17$

Cell Research (2009) 19:208-215. doi: 10.1038/cr.2008.320; published online 16 December 2008

\section{Introduction}

Interleukin-17 (IL-17) was originally isolated from an activated T-cell hybridoma in 1993 [1], and consists of an interleukin family with five other members (IL17B-F) that had been identified subsequently [2-4]. IL17 is mainly secreted by $\mathrm{T}_{\mathrm{H}} 17$ lymphocytes, a novel subset of $\mathrm{CD}^{+} \mathrm{T}$ helper cells that play a pivotal role in mediating autoimmunity and inflammation [5-12]. These $\mathrm{T}_{\mathrm{H}} 17$ lymphocytes have been shown to contribute to the pathogenesis of several autoimmune and inflammatory diseases, such as experimental autoimmune encephalitis (EAE) and collagen-induced arthritis (CIA) [7, 8], and consistent with this suggestion, IL-17-deficient mice are resistant to EAE and CIA [13]. IL-17 treatment primarily induces the expression of three classes of genes: (a)

Correspondence: Zhijie Chang

Tel: +86-10-62785076; Fax: +86-10-62773624

E-mail: zhijiec@tsinghua.edu.cn

Received 17 December 2007; revised 4 May 2008; accepted 8 July 2008; published online 16 December 2008 cytokines/chemokines, including IL-6, IL-8, CXCL1, CXCL2, CXCL5, CCL2, CCL5, CCL7 and CCL20; (b) cytoplasmic proteins involved in the immune response/ inflammation, including LCN2/24p3, COX2 and EREG; and (c) extracellular matrix/adherence proteins, including Mmp3, Mmp9, Mmp13 and ICAM-1 [2-4, 10, 14, 15] (also see review [16]). Recently, IL-17 was reported to induce the expression of RGD13 and RGS16, which, together with Cxcr4, regulate B-cell differentiation [17]. IL-17 also induces high levels of hBD-2 and IL-19 expression in human airway epithelial cells [18]. As IL-17 signaling is operative in many tissues, such as the brain, lung, articular cartilage, bone and intestine [2-4], it is clear that a major role of IL-17 is to regulate the cytokine network and provide a link between $\mathrm{T}_{\mathrm{H}} 17$ lymphocyte activation and the homeostasis of other tissues in health and disease.

IL-17 receptor (IL-17R or IL-17RA) is the founding member of the IL-17R family, which consists of five members, which have been known as IL-17RA-E to date [2-4]. Mice deficient in IL-17R fail to respond to IL-17 $[19,20]$, and soluble IL-17R or antibodies against IL$17 \mathrm{R}$ block the activity of IL-17 in human epithelial cells 
[21], showing that IL-17R is essential for IL-17 signaling.

At the time of discovery, IL-17 and IL-17R appeared striking in their uniqueness. IL-17 and IL-17R showed no clear similarity to any other known interleukin or cytokine receptor, nor did they even contain a known recognizable protein domain [4]. Thus, the IL-17 system seems to be a distinct signaling system differing from those of the other interleukins, explaining why IL-17 signaling cascades have remained elusive even after more than 10 years since their discovery. Recently, the identification of IL-17F and the analysis of its crystallographic structure have revealed that the homodimeric cytokines IL-17 and IL-17F share a similar structural feature with the cystine-knot growth factor family, suggesting that IL17 may have a relationship with these growth factors [22]. The cystine-knot growth factor superfamily is quite diverse, consisting of bone morphogenetic proteins (BMPs), transforming growth factor beta (TGF- $\beta$ ), platelet-derived growth factors (PDGFs), nerve growth factor (NGF) and other neurotrophins [23]. Members of the cystineknot growth factor superfamily function as a homodimer and bind both homodimeric and heteromeric receptors during signal transduction [23, 24]. On the basis of the structural information and the shared binding features with cystine-knot growth factors, we speculated that IL17 might signal through a heteromeric receptor complex. This hypothesis has been supported by the recent finding that IL-17RC is an essential component of the IL-17R signaling complex [19].

Subsequent to the discovery of IL-17RD (Sef), a SEFIR domain (after Sef and IL-17R's) was identified in transmembrane IL-17R's as well as other soluble factors such as Act1 [25]. Interestingly, it was recently reported that Act1 and IL-17R exhibit homotypic association mediated by the SEFIR domain and that Actl functions as an adaptor to mediate IL-17 signaling [26, 27]. In this study, we show that IL-17RD (Sef), an orphan receptor member of the IL-17R family earlier identified by our group and others [28-33], interacts with IL-17R and mediates IL-17 signaling. Our results provide novel evidence that IL-17 signals through a heteromeric and/or homomeric receptor complex that includes IL-17RD.

\section{Results}

\section{IL-17RD associates with IL-17R}

To identify additional members of the IL-17R family, we screened the National Center for Biotechnology Information (NCBI) database using the intracellular domain of IL-17R and cloned two isoforms of IL-17RD, IL-17RLM-L (Sef) and IL-17RLM-S (Sef-S) [29, 31].
We noted that IL-17RD (Sef), IL-17R and Act1 share the SEFIR domain [25]. Recently, Act1 has been shown to homotypically interact with IL-17R, mediated by the SEFIR domain, and to function as an adaptor protein for IL17 signaling $[26,27]$. We therefore speculated that IL17RD might also associate with IL-17R and mediate IL17 signaling.

To test this hypothesis, we first examined whether IL17RD interacts with IL-17R in cultured cells. When IL17RD and IL-17R were co-expressed in HEK293T cells, IL-17R was immunoprecipitated with IL-17RD and IL17 treatment did not enhance this association (Figure 1A). We further examined the interaction between the endogenous IL-17R and IL-17RD by precipitating the receptor complex with either anti-IL-17R or anti-IL-17RD antibody in either MEF or NIH3T3 cells. The results showed that the anti-IL-17R antibody co-precipitated the endogenous IL-17RD protein and vice versa (Figure 1B). Our data also indicate that the interaction of endogenous IL-17R with IL-17RD does not respond to IL-17 stimulation as there was no significant change in the intensity of the interaction in the presence or absence of IL-17 in both cell lines (Figure 1B, compare lanes 2 with 3, and 5 with 6). Immunoprecipitation experiments using extracts with or without overexpression of IL-17R or IL-17RD consistently indicate that IL-17R interacts with IL-17RD independently of IL-17 stimulation.

In addition, we used rabbit anti-IL-17RD serum to immunoprecipitate endogenous IL-17RD in HEK293T cells and showed the presence of IL-17R-Myc protein in the precipitated complexes (Figure 1C, lane 2). When IL-17RD was co-expressed, a greater amount of IL-17RMyc protein was co-precipitated (Figure 1C, lane 5 compared with lane 2), suggesting that both the endogenous IL-17RD and the overexpressed IL-17RD proteins associate with the IL-17R-Myc protein. Interestingly, we also found that IL-17RD interacts with IL-17RB (Figure 1C, lanes 3 and 6). Furthermore, we generated two mutants of IL-17RD, IL-17RD $\Delta$ ECD and IL-17RD $\Delta I C D$, which lack the extracellular domain (ECD) and the intracellular domain (ICD), respectively. Immunoprecipitation assay results with these mutants show that they are able to interact with IL-17R (Figure 1D), suggesting that the ECD or ICD alone is sufficient to establish an association between IL-17RD and IL-17R. These data also indicate that the association between these two molecules is not mediated only by SEFIR domain homotypic interaction, but that other domains may also contribute to the interactions.

\section{IL-17RD colocalizes with IL-17R}

Given that IL-17RD interacts with IL-17R, we further 


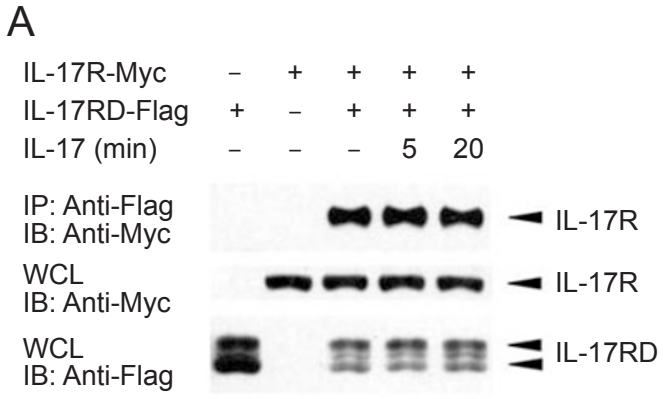

B

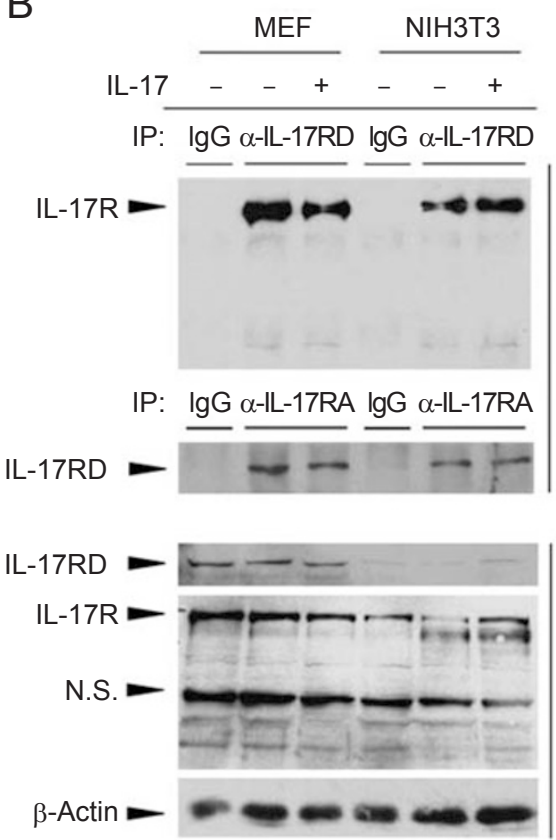<smiles>C1CCCCC1</smiles>
C IL-17RD $-\ldots+++$
IL-17R-Myc - + - +
IL-17RB-Myc - - + - +

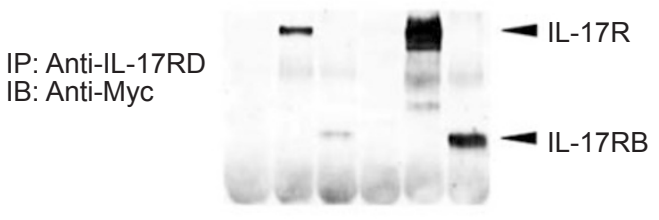

WCL

IB: Anti-Myc

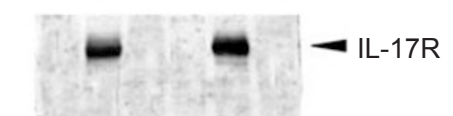

WCL

IB: Anti-IL-17RD

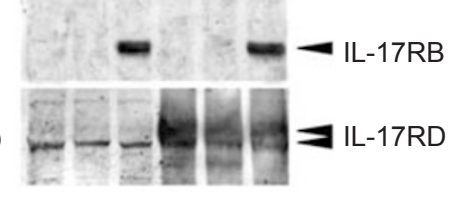

D

$\begin{array}{cl} & \text { IL-17R-HA } \\ & \text { IL-17RD-Myc } \\ & \text { IL-17RD } \Delta \text { ECD-Myc } \\ 0 & \text { IL } \\ \frac{\pi}{5} & \text { IL-17RD } \Delta \text { ICD-Myc } \\ \frac{0}{0} & \text { IP: Anti-Myc } \\ \frac{0}{0} & \text { IB: Anti-HA } \\ & \text { WCL } \\ & \text { IB: Anti-HA } \\ & \\ & \\ \text { IP: Anti-Myc } \\ \text { IB: Anti-Myc }\end{array}$

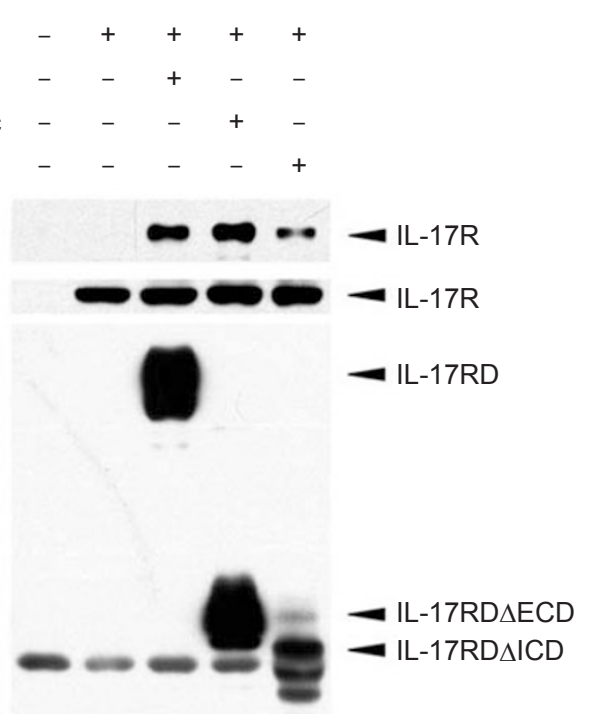

Figure 1 IL-17RD interacts with IL-17R. (A) IL-17R-Myc and IL-17RD-Flag interact in HEK293T cells. HEK293T cells were transfected with the indicated plasmids for $24 \mathrm{~h}$, treated with $100 \mathrm{ng} / \mathrm{ml} \mathrm{IL-17}$ for the indicated time, then lysed and immunoprecipitated with Flag antibody and Protein G PLUS-Agarose beads. Top panel, Flag immunoprecipitates were resolved by SDSPAGE and blotted with anti-Myc antibody to detect co-precipitated IL-17R. Center panel and bottom panel, whole cell lysates (WCL) of transfected cells were blotted with anti-Myc and anti-Flag antibodies to determine the expression levels of the IL-17R and IL-17RD proteins, respectively. (B) Interaction of endogenous IL-17R and IL-17RD. MEF and NIH3T3 cells treated with or without IL-17 stimulation were used to immunoprecipitate complexes after lysis. Anti-IL-17R and IL-17RD antibodies were used for immunoprecipitation and western blot analysis. $5 \%$ of the cell lysates were used for the input. (C) IL-17RD interacts with both IL-17R-Myc and IL-17RB-Myc. Immunoprecipitations were carried out using anti-IL-17RD rabbit polyclonal antibodies and protein G PLUS-Agarose beads and subjected to immunoblotting with anti-Myc antibody. (D) Both the intracellular and extracellular domains of IL-17RD interact with IL-17R. Immunoprecipitations were carried out using anti-Myc antibody and protein G PLUS-Agarose beads and subjected to immunoblotting with anti-HA antibody. IL-17RD $\triangle E C D$ represents the deletion of the extracellular domain of IL-17RD, and IL-17RD $\triangle I C D$ represents the deletion of the intracellular domain.

examined whether these two molecules colocalize within the cell. For this, we co-expressed Myc-tagged IL-17RD and HA-tagged IL-17R in COS7 cells and carried out immunostaining assays. The results show that when transfected alone, IL-17R showed a distribution primarily on the cell surface (Figure 2A, arrow indicated). In- terestingly, when IL-17RD, which exhibits a vesicle-like punctated staining pattern $[29,30]$, was co-expressed, IL-17R distribution changed into a punctated pattern colocalizing with IL-17RD (Figure 2B). These results indicate that IL-17RD is able to affect IL-17R localization, suggesting that these two molecules are colocalized and 

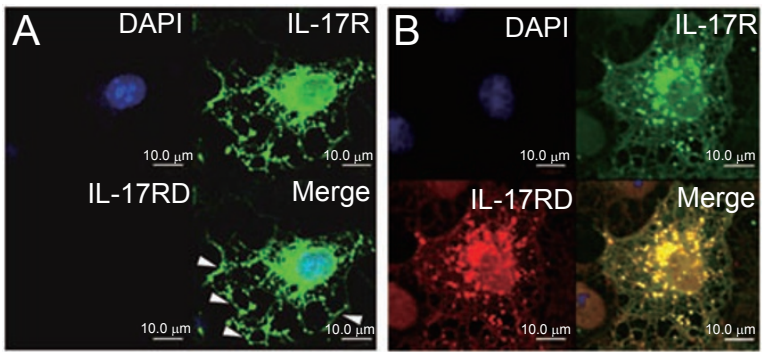

Figure 2 IL-17RD colocalizes with IL-17R. COS7 cells were grown on glass coverslips and transfected with $1.0 \mu \mathrm{g} \mathrm{IL-17R-}$ HA (A) or $1.0 \mu \mathrm{g}$ IL-17RD-Myc and $1.0 \mu \mathrm{g}$ IL-17R-HA (B). After $24 \mathrm{~h}$, cells were fixed and immunostained with mouse anti-Myc antibody, followed by TRITC-conjugated secondary antibody (red) and rabbit anti-HA antibody, followed by FITC-conjugated secondary antibody (green). Nuclei were counterstained with DAPI. Scale bar: $10.0 \mu \mathrm{m}$. associate with each other within cells.

\section{IL-17RD mediates IL-17 signaling}

To determine whether IL-17RD mediates IL-17 signaling, we used a luciferase reporter driven by the native promoter of mouse $24 \mathrm{p} 3$ as described earlier [34]. This reporter plasmid was transfected into mouse embryo fibroblasts (MEFs) together with IL-17RD and/or IL-17R. These experiments demonstrate that overexpression of IL-17RD or IL-17R enhances reporter activity in the absence of ligand. On IL-17 treatment, the IL-17RD- or IL17R-induced luciferase activity increased (Figure 3A). In addition, we found that IL-17RD is homomultimerized in the absence of ligand (data not shown), reminiscent of an earlier report showing that IL-17R self-associates independently of ligand stimulation [35]. These observations

$$
\text { A }
$$

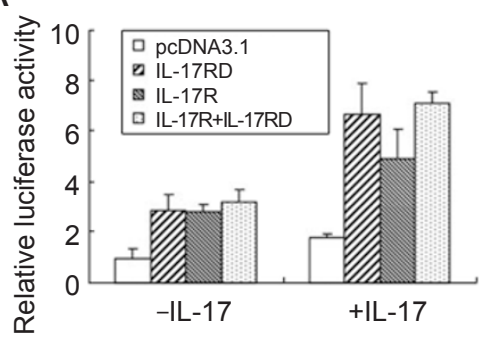

C
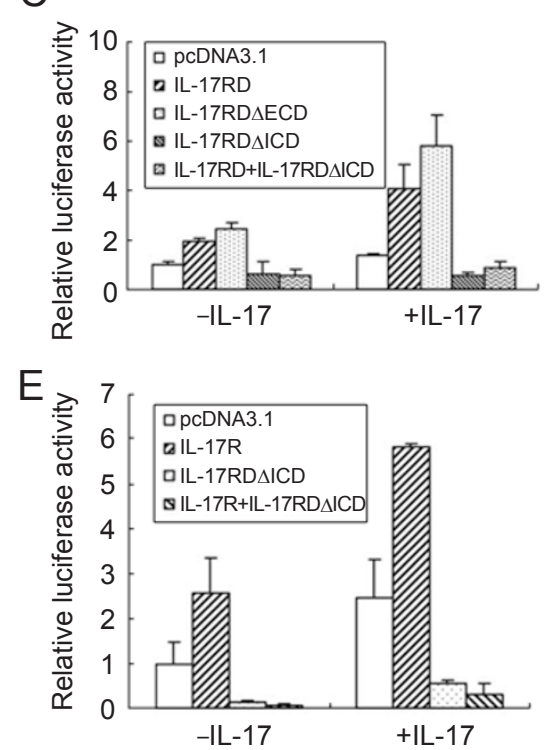

B
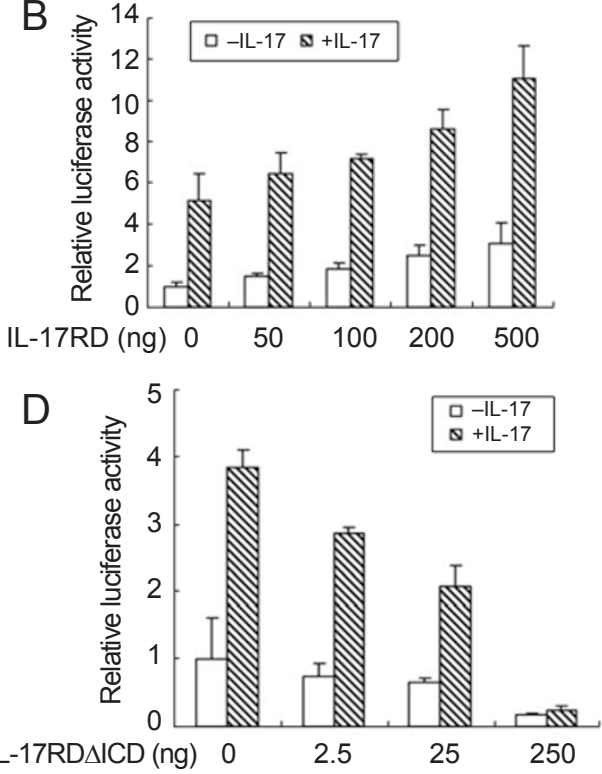

Figure 3 IL-17RD mediates IL-17 signaling. MEFs were transfected with 24p3 native promoter-luciferase reporter and IL17RD and/or IL-17R (A) or multiple doses of IL-17RD (B) or wild-type and mutant IL-17RD (C) or multiple doses of IL-17RD$\triangle \mathrm{ICD}(\mathrm{D})$ or IL-17RD $\triangle \mathrm{ICD}$ and/or IL-17R (E) plasmids as indicated. After $24 \mathrm{~h}$, cells were stimulated with (+IL-17) or without $(-\mathrm{IL}-17) 100 \mathrm{ng} / \mathrm{ml} \mathrm{IL-17}$ for $8 \mathrm{~h}$ and then luciferase activity was analyzed using the Dual-Luciferase Reporter Assay System. Data were normalized by co-transfection with a Renilla reniformis luciferase reporter vector. Results presented are from one experiment assayed in triplicate. Similar results were obtained in three independent experiments. 
indicate that forced expression of the receptors results in homodimerization and activation of downstream signaling, which is a known typical feature of type 1 receptors [36]. We also observed that the co-expression of IL17RD and IL-17R did not lead to any further increase in luciferase activity compared with single receptor expression (Figure 3A). A possible explanation for this observation is that additional receptor components, such as IL-17RC [19], may be limiting. Furthermore, dosedependent assay results showed that increasing amounts of IL-17RD led to higher luciferase activity (Figure 3B), supporting the fact that IL-17RD is involved in IL-17 signaling.

To determine which domain of IL-17RD is involved in IL-17 signaling, we co-transfected IL-17RD mutants and the reporter plasmid into MEFs. Luciferase assays demonstrated that IL-17RD $\triangle \mathrm{ECD}$ retains the ability to mediate IL-17 signaling, whereas IL-17RD $\triangle$ ICD fails to do so (Figure 3C). As IL-17RD $\triangle \mathrm{ICD}$ does not deliver the IL-17 signal (Figure 3C) but still interacts with IL17R (Figure 1C), we speculated that IL-17RD $\Delta \mathrm{ICD}$ might function as a dominant-negative form. To test this hypothesis, we carried out luciferase reporter experiments and found that when it was co-expressed with IL17RD, IL-17RD $\triangle \mathrm{ICD}$ blocked IL-17RD-mediated IL17 signaling (Figure 3C, the last columns in each group). Furthermore, dose-dependent assay results showed that increasing amounts of IL-17RD $\triangle \mathrm{ICD}$ led to lower activity of the reporter (Figure 3D). Interestingly, when IL17RD $\Delta \mathrm{ICD}$ was co-expressed with IL-17R, luciferase activity was also inhibited, suggesting that this dominantnegative mutant also suppresses IL-17R-mediated IL-17 signaling (Figure 3E). These novel observations indicate that IL-17RD plays an important role in the IL-17 signaling pathway.

\section{IL-17RD physically associates with TRAF6}

As the intracellular domain of IL-17RD is pivotal

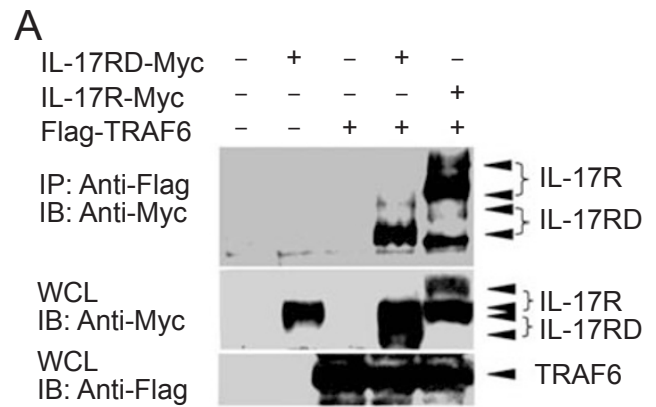

to its function, we tested whether IL-17RD associates physically with molecules downstream of IL-17R. Given that TRAF6 has been shown to interact with IL-17R and is essential for IL-17 signaling [34, 37], we co-expressed IL-17RD and TRAF6 in HEK293T cells and examined their potential interaction by immunoprecipitation assays. Our results showed that IL-17RD was co-immunoprecipitated with TRAF6 and vice versa (Figure 4A and 4B), indicating that IL-17RD and TRAF6 are capable of associating with each other.

\section{Discussion}

$\mathrm{T}_{\mathrm{H}} 17$ cells are a new type of effector $\mathrm{T}$ cells that have been discovered recently. Most current research on $T_{H} 17$ cells has focused on the mechanisms of $T_{H} 17$ cell differentiation $[38,39]$, whereas relatively little is known about how IL-17 exerts its effects on target tissues. In this study, we show that IL-17RD colocalizes and associates with IL-17R to mediate IL-17 signaling. The precise composition of the IL-17R complex on the cell membrane and how this complex mediates signaling are still not fully understood (see review [16]); thus, our study provides novel evidence that IL-17R forms a heteromeric complex with IL-17RD. Indeed, it has been reported earlier that IL-17R associates with IL-17RC [19]. More interestingly, we identified that IL-17RD associates not only with IL-17R but also with IL-17RB (see Figure $1 \mathrm{C})$. These data suggest that IL-17R family members may form different receptor complexes to allow interaction with different ligands. It has been reported that IL$17 \mathrm{~A}$ and IL-17F form a heterodimer in activated human $\mathrm{CD}^{+} \mathrm{T}$ cells [40], implying the possibility that different ligands and receptors may be composed at the cell membrane under different physiological conditions.

The fact that IL-17RD $\triangle I C D$ is unable to mediate IL17 signaling but functions as a dominant-negative form (Figure 3C-E) indicates that the intracellular domain of

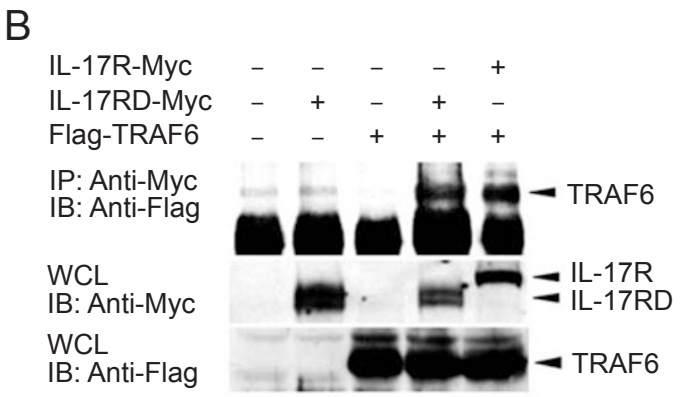

Figure 4 IL-17RD physically associates with TRAF6. The procedures were similar to those described in Figure 1. Immunoprecipitations were carried out using anti-Flag (A) or anti-Myc (B) antibodies and protein G PLUS-Agarose beads and subjected to immunoblotting with anti-Myc (A) or anti-Flag (B) antibodies, respectively. 
IL-17RD is pivotal. In addition, IL-17RD interacts with the IL-17R downstream molecule TRAF6 (Figure 4A and 4B). We therefore propose that the IL-17RD intracellular domain interacts with IL-17R and TRAF6 to deliver the downstream signal. Act1 has recently been reported to be an adaptor protein of IL-17R and to mediate immediate receptor downstream signaling [26, 27]; therefore, it should be very interesting to investigate the interactions and functional relationships among IL-17R, IL-17RD, Act1 and TRAF6, especially in light of our earlier results showing that TRAF6 can mediate IL-17R ubiquitination [34].

IL-17R is ubiquitously expressed; thus, most cells are potential targets of IL-17 action [2]. A major role of IL-17 and $\mathrm{T}_{\mathrm{H}} 17$ cells has been described in immunemediated injury models, such as organ-specific autoimmunity in the brain, heart, synovium and intestines, allergic disorders of the lung and skin, and microbial infections of the intestines and the nervous system [5]. The expression pattern of hIL-17RD (hSef) has been examined by northern blotting [41, 42], and interestingly, the hIL-17RD transcript shows the highest expression in the kidney, followed by the heart, small intestine, colon, skeletal muscle, brain, lung and spleen, but with barely detectable expression in the thymus, liver, placenta and peripheral blood leukocytes. Considering the expression patterns of IL-17R and IL-17RD, and the tissues where IL-17 and $\mathrm{T}_{\mathrm{H}} 17$ execute their functions, it is reasonable to suggest that IL-17RD plays a role in IL-17 signaling in cognate tissues.

IL-17RD has recently been shown to inhibit FGF signaling [28-33] and to facilitate EGF signaling [43]. In this report, we provide data showing that IL-17RD functions in IL-17 signaling, suggesting that IL-17RD has two distinct types of functions. IL-17RD-deficient mice do not show any obvious phenotype related to FGF signaling [44]; thus, it should be interesting to examine whether these knock-out mice have a phenotype similar to that of IL-17R-deficient mice when challenged with specific pathogens, such as Klebsiella pneumoniae [20]. These types of assays should provide interesting information to further our understanding of the functions of IL-17RD in IL-17 signaling in vivo.

In conclusion, we have shown that IL-17RD colocalizes and associates with IL-17R and mediates IL-17 signaling possibly through its intracellular domain.

\section{Materials and Methods}

\author{
Plasmid construction \\ pcDNA3/hIL-17RD (pcDNA3/hSef), pcDNA3.1/Myc-His/hIL- \\ 17RD (pcDNA3.1/Myc-His/hSef) and pcDNA3.1/Myc-His/hIL-
}

17R plasmids were described earlier [29, 31, 34]. pcDNA3.1/MycHis/hIL-17RD $\triangle I C D$, an IL-17RD mutant lacking the intracellular domain, was constructed by standard polymerase chain reaction (PCR) mutagenesis using the following primers: forward primer, 5'-tat aag ctt gec gcc acc atg gec ccg tgg ctg-3'; reverse primer, $5^{\prime}$-att ctc gag cat cac agt gaa gag cg-3'. pcDNA3.1/Myc-His/sphIL-17RD $\triangle E C D$, used for expression of an IL-17RD mutant lacking the extracellular domain, was constructed with pcDNA3.1/ Myc-His/sp, which was modified from pcDNA3.1/Myc-His as described earlier [29] by inserting a fragment amplified by PCR using the following primers: forward primer, $5^{\prime}$-gca tga att ccc cat cag agc cgt g-3'; reverse primer, 5'-att ctc gag caa agg ggc gac cgc3'. pcDNA3.1/HA-His/hIL-17R was subcloned from pcDNA3.1/ Myc-His/hIL-17R by double digestion using HindIII and EcoRI restriction endonucleases. PC4-hIL-17RB plasmid was provided by Dr Reinhard Ebner (Human Genome Science, Inc., Rockville, MD) [45]. The coding sequence of IL-17RB was amplified from this construct using the following primers: forward primer, 5'tag aat tcg cca cca tgt cgc tcg tgc tgc t-3'; reverse primer, 5'-gat ctc gag aag gca agg gga aga gtc $\mathrm{t}-3^{\prime}$, and then inserted into the HindIII/XhoI sites of pcDNA3.1/Myc-His vector. All of these constructs were confirmed by sequencing. Flag-TRAF6 was provided by Dr Hiroyasu Nakano (Juntendo University, Tokyo, Japan).

\section{Cell culture and transfection}

HEK293T cells, COS7 cells, NIH3T3 cells and MEFs were cultured in DMEM containing $10 \% \mathrm{FBS}$ at $37{ }^{\circ} \mathrm{C}$ in $5 \% \mathrm{CO}_{2}-$ containing atmosphere. Transfection was performed by using CalPhos Mammalian Transfection Kit (Clontech) for HEK293T cells, lipofect AMINE2000 (Invitrogen) for COS7 cells and High Efficient Transfection Reagent (Vigorous) for MEFs and NIH3T3 cells according to the manufacturer's protocols. The NIH3T3-IL17RD stable cell line was established by selection with G418 from the NIH3T3 cells transfected with pcDNA3.1/Myc-His/hSef [29].

\section{Immunoprecipitation and immunoblotting}

Immunoprecipitation from transfected cells was done as described earlier [29]. Anti-Myc (9E10, mouse, Santa Cruz Biotechnology), anti-HA (F-7, mouse, Santa Cruz Biotechnology), antiFlag (M2, mouse, Sigma) and anti-IL-17RD antibodies were used in the experiments. Anti-IL-17RD (anti-Sef) rabbit polyclonal antibody has been described earlier [31]. Anti-IL-17R and anti-IL17RD antibodies and IL-17 were purchased from R\&D Systems.

\section{Luciferase assays}

The construction of the $24 \mathrm{p} 3$ promoter-luciferase reporter $(-1385$ to +1$)$ was described earlier [34]. Luciferase assays were carried out in MEFs by co-transfecting $24 \mathrm{p} 3$ promoter-luciferase plasmid $(2.0 \mu \mathrm{g}$ for a 24 -well plate) and different constructs as indicated. After $24 \mathrm{~h}$, the cells were stimulated with $100 \mathrm{ng} / \mathrm{ml} \mathrm{IL-17}$ (17-IL, R\&D Systems) for $8 \mathrm{~h}$. Luciferase activity was measured by the Dual Luciferase Assay System (Promega) using Top Count (Packard). The Renilla reniformis luciferase plasmid (pRL-TK, Promega) was co-transfected as an internal control. Experiments were carried out in triplicate for each treatment and the average and standard deviations are shown.

\section{Immunofluorescent staining}

COS7 cells were fixed $24 \mathrm{~h}$ after transfection, with $4 \%$ para- 
formaldehyde for $20 \mathrm{~min}$, perforated with $0.3 \%$ Triton X-100 for $10 \mathrm{~min}$, and then the cells were blocked with $10 \%$ FBS for $50 \mathrm{~min}$, followed by incubation with mouse Myc (9E10, Santa Cruz Biotechnology) and rabbit HA (Y-11, Santa Cruz Biotechnology) antibodies at $4{ }^{\circ} \mathrm{C}$ overnight. Cells were incubated with the secondary antibodies, goat anti-mouse IgG/TRITC and goat anti-rabbit IgG/ FITC (Jackson Research Laboratories), for $1 \mathrm{~h}$ and counterstained with DAPI for $5 \mathrm{~min}$. Finally, the immunostained cells were visualized with a confocal laser scanning microscope (OLYMPUS BX61).

\section{Acknowledgments}

We thank Dr Xin-Yuan Fu (Indiana University, Indianapolis, USA) for his strong support and suggestions on this project. We are grateful to Drs Reinhard Ebner (Human Genome Science, Inc., Rockville, MD) and Hiroyasu Nakano (Juntendo University, Tokyo, Japan) for providing constructs. This work was supported by a Tsinghua-Yu-Yuan Medical Sciences Fund and grants from the National Natural Science Foundation of China (No. 30530420, 30470888 and 30518002), Chinese National Support Project (2006CB910102) and 973 Project (2002CB513007) to Zhijie Chang, and a China-Canada Joint Health Research Initiative grant to Jim Hu (\#CCI-82411) and Zhijie Chang (\#30611120522). We thank Dr David M Irwin (University of Toronto, Toronto, Canada) for reading the paper.

\section{References}

1 Rouvier E, Luciani MF, Mattei MG, Denizot F, Golstein P. CTLA-8, cloned from an activated $\mathrm{T}$ cell, bearing AU-rich messenger RNA instability sequences, and homologous to a herpesvirus saimiri gene. J Immunol 1993; 150:5445-5456.

2 Kolls JK, Linden A. Interleukin-17 family members and inflammation. Immunity 2004; 21:467-476.

3 Moseley TA, Haudenschild DR, Rose L, Reddi AH. Interleukin-17 family and IL-17 receptors. Cytokine Growth Factor Rev 2003; 14:155-174.

4 Aggarwal S, Gurney AL. IL-17: prototype member of an emerging cytokine family. J Leukoc Biol 2002; 71:1-8.

5 Steinman L. A brief history of $\mathrm{T}(\mathrm{H}) 17$, the first major revision in the $\mathrm{T}(\mathrm{H}) 1 / \mathrm{T}(\mathrm{H}) 2$ hypothesis of $\mathrm{T}$ cell-mediated tissue damage. Nat Med 2007; 13:139-145.

6 Weaver CT, Hatton RD, Mangan PR, Harrington LE. IL-17 family cytokines and the expanding diversity of effector $\mathrm{T}$ cell lineages. Annu Rev Immunol 2007; 25:821-852.

7 Weaver CT, Harrington LE, Mangan PR, Gavrieli M, Murphy KM. Th17: an effector CD4 T cell lineage with regulatory $\mathrm{T}$ cell ties. Immunity 2006; 24:677-688.

8 Dong C. Diversification of T-helper-cell lineages: finding the family root of IL-17-producing cells. Nat Rev Immunol 2006; 6:329-333.

9 Harrington LE, Hatton RD, Mangan PR, et al. Interleukin 17-producing CD4+ effector $\mathrm{T}$ cells develop via a lineage distinct from the T helper type 1 and 2 lineages. Nat Immunol 2005; 6:1123-1132.

10 Park H, Li Z, Yang XO, et al. A distinct lineage of CD4 T cells regulates tissue inflammation by producing interleukin 17. Nat Immunol 2005; 6:1133-1141.
11 Langrish CL, Chen Y, Blumenschein WM, et al. IL-23 drives a pathogenic $\mathrm{T}$ cell population that induces autoimmune inflammation. J Exp Med 2005; 201:233-240.

12 Aggarwal S, Ghilardi N, Xie MH, de Sauvage FJ, Gurney AL. Interleukin-23 promotes a distinct CD4 $\mathrm{T}$ cell activation state characterized by the production of interleukin-17. J Biol Chem 2003; 278:1910-1914.

13 Nakae S, Nambu A, Sudo K, Iwakura Y. Suppression of immune induction of collagen-induced arthritis in IL-17-deficient mice. J Immunol 2003; 171:6173-6177.

14 Shen F, Ruddy MJ, Plamondon P, Gaffen SL. Cytokines link osteoblasts and inflammation: microarray analysis of interleukin-17- and TNF-alpha-induced genes in bone cells. J Leukoc Biol 2005; 77:388-399.

15 Ruddy MJ, Shen F, Smith JB, Sharma A, Gaffen SL. Interleukin-17 regulates expression of the CXC chemokine LIX/ CXCL5 in osteoblasts: implications for inflammation and neutrophil recruitment. J Leukoc Biol 2004; 76:135-144.

16 Shen F, Gaffen SL. Structure-function relationships in the IL17 receptor: implications for signal transduction and therapy. Cytokine 2008; 41:92-104.

17 Hsu HC, Yang P, Wang J, et al. Interleukin 17-producing T helper cells and interleukin 17 orchestrate autoreactive germinal center development in autoimmune BXD2 mice. Nat Immunol 2008; 9:166-175.

18 Huang F, Kao CY, Wachi S, et al. Requirement for both JAKmediated PI3K signaling and ACT1/TRAF6/TAK1-dependent NF-kappaB activation by IL-17A in enhancing cytokine expression in human airway epithelial cells. J Immunol 2007; 179:6504-6513.

19 Toy D, Kugler D, Wolfson M, et al. Cutting edge: interleukin 17 signals through a heteromeric receptor complex. J Immunol 2006; 177:36-39.

20 Ye P, Rodriguez FH, Kanaly S, et al. Requirement of interleukin 17 receptor signaling for lung $\mathrm{CXC}$ chemokine and granulocyte colony-stimulating factor expression, neutrophil recruitment, and host defense. J Exp Med 2001; 194:519-527.

21 McAllister F, Henry A, Kreindler JL, et al. Role of IL-17A, IL-17F, and the IL-17 receptor in regulating growth-related oncogene-alpha and granulocyte colony-stimulating factor in bronchial epithelium: implications for airway inflammation in cystic fibrosis. J Immunol 2005; 175:404-412.

22 Hymowitz SG, Filvaroff EH, Yin JP, et al. IL-17s adopt a cystine knot fold: structure and activity of a novel cytokine, IL-17F, and implications for receptor binding. EMBO J 2001; 20:5332-5341.

23 McDonald NQ, Hendrickson WA. A structural superfamily of growth factors containing a cystine knot motif. Cell 1993; 73:421-424.

$24 \mathrm{Lu} \mathrm{B}$, Pang PT, Woo NH. The yin and yang of neurotrophin action. Nat Rev Neurosci 2005; 6:603-614.

25 Novatchkova M, Leibbrandt A, Werzowa J, Neubuser A, Eisenhaber F. The STIR-domain superfamily in signal transduction, development and immunity. Trends Biochem Sci 2003; 28:226-229.

26 Qian Y, Liu C, Hartupee J, et al. The adaptor Act1 is required for interleukin 17-dependent signaling associated with autoimmune and inflammatory disease. Nat Immunol 2007; 8:247-256.

27 Chang SH, Park H, Dong C. Act1 adaptor protein is an im- 
mediate and essential signaling component of interleukin-17 receptor. J Biol Chem 2006; 281:35603-35607.

28 Ren Y, Li Z, Rong Z, et al. Tyrosine 330 in hSef is critical for the localization and the inhibitory effect on FGF signaling. Biochem Biophys Res Commun 2007; 354:741-746.

29 Rong Z, Ren Y, Cheng L, et al. Sef-S, an alternative splice isoform of sef gene, inhibits NIH3T3 cell proliferation via a mitogen-activated protein kinases p42 and p44 (ERK1/2)independent mechanism. Cell Signal 2007; 19:93-102.

30 Ren Y, Cheng L, Rong Z, et al. hSef co-localizes and interacts with Ras in the inhibition of Ras/MAPK signaling pathway. Biochem Biophys Res Commun 2006; 347:988-993.

31 Xiong S, Zhao Q, Rong Z, et al. hSef inhibits PC-12 cell differentiation by interfering with Ras-mitogen-activated protein kinase MAPK signaling. J Biol Chem 2003; 278:50273-50282.

32 Tsang M, Friesel R, Kudoh T, Dawid IB. Identification of Sef, a novel modulator of FGF signalling. Nat Cell Biol 2002; 4:165-169.

33 Furthauer M, Lin W, Ang SL, Thisse B, Thisse C. Sef is a feedback-induced antagonist of Ras/MAPK-mediated FGF signalling. Nat Cell Biol 2002; 4:170-174.

34 Rong Z, Cheng L, Ren Y, et al. Interleukin-17F signaling requires ubiquitination of interleukin-17 receptor via TRAF6. Cell Signal 2007; 19:1514-1520.

35 Kramer JM, Yi L, Shen F, et al. Evidence for ligand-independent multimerization of the IL-17 receptor. J Immunol 2006; 176:711-715.

36 Lemmon MA, Schlessinger J. Regulation of signal transduction and signal diversity by receptor oligomerization. Trends
Biochem Sci 1994; 19:459-463.

37 Schwandner R, Yamaguchi K, Cao Z. Requirement of tumor necrosis factor receptor-associated factor (TRAF)6 in interleukin 17 signal transduction. J Exp Med 2000; 191:1233-1240.

38 Dong C. TH17 cells in development: an updated view of their molecular identity and genetic programming. Nat Rev Immunol 2008; 8:337-348.

39 McGeachy MJ, Cua DJ. Th17 cell differentiation: the long and winding road. Immunity 2008; 28:445-453.

40 Wright JF, Guo Y, Quazi A, et al. Identification of an interleukin $17 \mathrm{~F} / 17 \mathrm{~A}$ heterodimer in activated human CD4+ T cells. $J$ Biol Chem 2007; 282:13447-13455.

41 Yang RB, Ng CK, Wasserman SM, et al. A novel interleukin-17 receptor-like protein identified in human umbilical vein endothelial cells antagonizes basic fibroblast growth factorinduced signaling. J Biol Chem 2003; 278:33232-33238.

42 Huang G, Xiong S, Zhao Q, et al. Expression of hSef in various human tissues and cell lines. Front Biol China 2006; 2:6.

43 Ren Y, Cheng L, Rong Z, et al. hSef potentiates EGF-mediated MAPK signaling through affecting EGFR trafficking and degradation. Cell Signal 2008; 20:518-533.

44 Lin W, Jing N, Basson MA, et al. Synergistic activity of Sef and Sprouty proteins in regulating the expression of Gbx2 in the mid-hindbrain region. Genesis 2005; 41:110-115.

45 Shi Y, Ullrich SJ, Zhang J, et al. A novel cytokine receptorligand pair. Identification, molecular characterization, and in vivo immunomodulatory activity. J Biol Chem 2000; 275:19167-19176. 